\title{
ROLE OF ADAPTIVE IMMUNITY IN \\ NERVOUS NECROSIS VIRUS PERSISTENT INFECTION ON GROUPER BY TRANSCRIPTOME APPROACH
}

\author{
Chun-Hsi Tso, Ming-Wei Lu ${ }^{\S}$ \\ Department of Aquaculture, National Taiwan Ocean University, Taiwan, 20224
}

\begin{abstract}
Viral Nervous Necrosis (VNN) is an important infectious fish disease which is caused by Nervous Necrosis Virus (NNV). NNV could infect more than 30 species freshwater and marine fish. In Taiwan, the major host for NNV infection is grouper and has caused severely economic loss. However, there is still deficient information about the mechanism of persistent infection during NNV infection. In this study, we used next generation sequencing (NGS) technique to investigate the differential expression between live NNV bathed and inactivated-NNV bathed grouper. The brain and head kidney of grouper were sampled on 10 days post infection. The RNA-seq analysis was performed on the basis of a de novo assembled transcriptome. The results showed the immune-related gene expression in brain was strongly up-regulated on persistent infection fish. Including signal transduction, regulation of DNA transcription, protein ubiquitination and apoptotic process were observed highly expression than inactivated-NNV bathed grouper brain. However, the expression of adaptive immune response on NNV persistent infection grouper head kidney was lower than inactivated-NNV bathed grouper. This might imply there is a regulation relationship between NNV and adaptive immune immunity on grouper.
\end{abstract}

\section{KEYWORDS}

RNA-Seq, Nerves Necrosis Virus, persistent infection, adaptive immunity, grouper

${ }^{\S}$ Corresponding author. Tel.: +886 24622192 \#5206

E-mail address: mingwei@ntou.edu.tw 